\title{
ACESSO À ASSISTÊNCIA PRÉ-NATAL NO BRASIL: ANÁLISE DOS DADOS DA PESQUISA NACIONAL DE SAÚDE
}

\section{Prenatal care access in Brazil: analysis of the National Health Research data Accesibilidad a la atención prenatal en Brasil: análisis de los datos de la Encuesta Nacional de Salud}

\author{
Aryelly Dayane da Silva Nunes \\ Universidade Federal do Rio Grande do Norte - UFRN - Natal (RN) - Brasil
}

\section{Ana Edimilda Amador}

Universidade Federal do Rio Grande do Norte - UFRN - Natal (RN) - Brasil

Ana Patrícia de Queiroz Medeiros Dantas

Universidade Federal do Rio Grande do Norte - UFRN - Natal (RN) - Brasil

Ulicélia Nascimento de Azevedo

Universidade Federal do Rio Grande do Norte - UFRN - Natal (RN) - Brasil

\section{Isabelle Ribeiro Barbosa}

Universidade Federal do Rio Grande do Norte - UFRN - Santa Cruz (RN) - Brasil

\section{RESUMO}

Objetivo: Analisar o acesso à assistência pré-natal no Brasil a partir dos dados da Pesquisa Nacional de Saúde (PNS) de 2013. Métodos: Estudo transversal com análise descritiva dos dados da PNS 2013, coletados no DATASUS entre dezembro de 2016 e janeiro de 2017. Responderam à pesquisa mulheres de 18 a 49 anos de idade que tiveram algum parto entre 01/01/2012 a 27/07/2013. A cobertura do pré-natal foi definida pela proporção de mulheres que responderam positivamente ao questionamento: "Na última vez que a senhora esteve grávida, a senhora fez pré-natal?". Os dados coletados foram: assistência pré-natal, idade, cor da pele, grau de instrução, aconselhamentos, recebimento do cartão pré-natal, consultas realizadas, assistência ao parto por região ou unidade da federação. Resultados: Houve elevada cobertura do acompanhamento pré-natal $(97,4 \%$ IC95\% 96,5-98,4), com entrega do cartão pré-natal $(95,3 \%$ IC95\% 93,6-97,0) e início de pré-natal com menos de 13 semanas de gestação $(83,7 \%$ IC95\% 80,7-86,6), porém baixa proporção de gestantes com todos os aconselhamentos $(69,2 \%$ IC95\% 65,5-72,9) e com exames para Sífilis $(64,8 \%$ IC95\% 61,0-68,7). Entre as que realizaram o pré-natal, 69,33\% das gestantes indicaram que todas as consultas foram feitas através do Sistema Único de Saúde. As regiões Norte e Nordeste apresentaram maior proporção de partos vaginais, porém menor número de consultas de pré-natal por gestante. Conclusão: Apesar de elevada cobertura da assistência pré-natal no Brasil, os indicadores mostram que ainda há inadequações no acesso ao serviço.

Descritores: Cuidado Pré-Natal; Saúde da Mulher; Acesso aos Serviços de Saúde; Avaliação de programas e projetos de saúde.

\begin{abstract}
Objective: To analyze the access to prenatal care in Brazil based on data from the 2013 National Health Survey (Pesquisa Nacional de Saúde-PNS). Methods: Cross-sectional study with descriptive analysis of the 2013 PNS data, retrieved from DATASUS between December 2016 and January 2017. Respondents to the survey were women aged 18 to 49 years who gave birth between 01/01/2012 and 07/27/2013. Prenatal coverage was defined by the proportion of women who responded positively to the question: "The last time you were pregnant, did you get prenatal care?" Data on prenatal care, age, skin color, education, counseling, receiving the prenatal care card, consultations held, and childbirth assistance by region or federation unit were collected from December 2016 to January 2017. Results: There was a high coverage of prenatal follow-up (97.4\% 95\% CI 96.5-98.4), with delivery of the prenatal card (95.3\% 95\% CI 93.6-97.0) and initiation under 13 gestational weeks (83.7\% 95\% CI 80.7-86.6), but a low proportion of pregnant women with all counseling (69.2\% CI 95\% 65.5-72.9) and syphilis tests (64.6\% 95\% CI 61.0-68.7). Among the pregnant women who underwent prenatal care, $69.33 \%$ indicated that all consultations were made through the Unified Health System. The North and Northeast Regions presented a higher proportion of vaginal delivery, but a lower number of prenatal consultations per pregnant woman. Conclusions: Despite the high coverage of prenatal care in Brazil, the indicators show that there are still inadequacies in the access to the service.
\end{abstract}

Descriptors: Prenatal care; Women's Health; Access to Health Services; Evaluation of health programs and projects. 


\section{RESUMEN}

Objetivo: Analizar la accesibilidad a la atención prenatal en Brasil con los datos de la Encuesta Nacional de Salud (ENS) de 2013. Métodos: Estudio transversal con análisis descriptivo de los datos de la ENS de 2013 recogidos del DATASUS entre diciembre de 2016 y enero de 2017. Contestaron a la encuesta las mujeres entre 18 y 49 años de edad que realizaron parto entre 01/01/2012 y 27/07/2013. La cobertura del prenatal ha sido definida por la proporción de mujeres que contestaron positivamente a la pregunta: "Has realizado el prenatal en su último embarazo?" Fueron recogidos datos de la atención prenatal, la edad, el color de la piel, el grado de escolaridad, los consejos recibidos, el recibimiento de la tarjeta prenatal, las consultas realizadas, la atención al parto por región o unidad de la federación. Resultados: Hubo elevada cobertura del seguimiento prenatal (97,4\% IC95\% 96,5-98,4), con la entrega de la tarjeta de prenatal (95,3\% IC95\% 93,6-97,0) y el inicio del prenatal con menos de 13 semanas de embarazo (83,7\% IC95\% 80,7-86,6), sin embargo, baja proporción de embarazadas ha recibido todos los consejos $(69,2 \%$ IC95\% 65,5-72,9) y tenía las pruebas de sifilis $(64,8 \%$ IC95\% 61,0-68,7). Entre las que han realizado el prenatal, el 69,33\% de las embarazadas indicó que todas las consultas han sido realizadas por el Sistema Único de Salud. Las regiones Norte y Noreste presentaron más proporción de partos vaginales pero menos cantidad de consultas de prenatal para cada embarazada. Conclusión: Pese a que haya una elevada cobertura de la atención prenatal en Brasil, los indicadores señalan que todavía hay inadecuaciones para el acceso al servicio.

Descriptores: Atención Prenatal; Salud de la Mujer; Accesibilidad a los Servicios de Salud; Evaluación de Programas y Proyectos de Salud.

\section{INTRODUÇÃO}

A cada minuto morre no mundo uma mulher em virtude de complicações da gravidez e do parto, e, para cada mulher que morre, outras trinta sofrem sequelas ou problemas crônicos de saúde ${ }^{(1,2)}$. Apesar do declínio estimado de $45 \%$ da mortalidade materna no mundo, há uma desproporção entre as taxas de mortalidade materna, sendo, em 2013, 14 vezes maiores em regiões em desenvolvimento do que em regiões desenvolvidas. Em 1990, o Brasil apresentava taxa de mortalidade materna de $120 / 100.000$ nascidos vivos (NV), de 85/100.000 NV em 2000 e 69/100.000 NV em 2013. O Brasil é classificado como "progredindo" em relação à melhoria da saúde materna pelo declínio anual da taxa de mortalidade materna ${ }^{(3)}$.

A redução da mortalidade materna figura entre os oito Objetivos de Desenvolvimento do Milênio, adotados pela Organização das Nações Unidas, com pactuação de redução de $75 \%$ até o ano de 2015, e, para atingir esta meta, seria necessário a redução de 5,5\% ao ano ${ }^{(4,5)}$. Apesar da considerável redução da mortalidade materna registrada no Brasil ao longo das últimas duas décadas $^{(6)}$, parece ter ocorrido uma estagnação na situação da mortalidade materna desde $2002^{(7)}$. Entre 1990 e 2013 , o país reduziu em $43 \%$ a taxa de mortalidade materna.

No final da primeira década dos anos 2000 , passou a existir um relativo consenso de que a mortalidade materna poderia ser reduzida através do fortalecimento dos sistemas de saúde para a oferta de serviços integrados, contínuos e de qualidade para mulheres durante e após a gestação, tanto na atenção de rotina quanto na emergência ${ }^{(1,5)}$.

A implantação do Programa de Humanização do Pré-Natal e Nascimento (PHPN) no ano de 2000, pelo Ministério da Saúde, e, em 2011, a instituição da Rede Cegonha, programa que estrutura e organiza a atenção à saúde materno-infantil no Brasil e tem como um de seus componentes a assistência pré-natal, tem impactado nos indicadores de acesso à assistência prénatal no país ${ }^{(1,8-10)}$. Para uma assistência pré-natal efetiva, é imprescindível a realização do número de consultas recomendadas, a identificação precoce de todas as gestantes na comunidade, o pronto início do acompanhamento pré-natal ainda no $1^{\circ}$ trimestre da gravidez, o cadastro da gestante no SIS- Pré- Natal, o fornecimento e preenchimento do cartão pré-natal, o esclarecimento sobre o calendário de vacinas e os exames preconizados, dentre outras ações de promoção da saúde ${ }^{(8,9)}$.

A avaliação da qualidade do pré-natal pode ser realizada por meio de critérios que julgam o acesso, embora devam considerar não somente o número de consultas, ou a idade gestacional de início do acompanhamento, mas a adequação do conteúdo da assistência prestada, além das desigualdades no acesso existentes entre as regiões brasileiras e entre os grupos populacionais ${ }^{(11)}$.

Todavia a realidade brasileira mostra que são registradas falhas na assistência pré-natal, tais como: dificuldades no acesso, início tardio, número inadequado de consultas e realização incompleta dos procedimentos preconizados, afetando sua qualidade e efetividade. A falta de vínculo entre os serviços que prestam a assistência pré-natal e ao parto é outro problema identificado, resultando na peregrinação da gestante em trabalho de parto na busca de uma vaga para internação, trazendo riscos adicionais à saúde da parturiente e do recém-nascido ${ }^{(12)}$.

De forma geral, os estudos mostram que houve aumento da cobertura da atenção pré-natal ao longo dos últimos dez anos em quase todo o país, podendo ultrapassar os $90 \%$ de cobertura, porém ainda persistem o elevado nível de inadequação e as marcantes disparidades regionais e sociais na cobertura e na qualidade dos serviços ofertados ${ }^{(12-17)}$. Por outro lado, os estudos têm divergido em relação à cobertura no acesso à assistência pré-natal no Brasil, de acordo com as regiões do estudo, pela diferença na população alvo analisada, além dos diferentes indicadores utilizados para mensurar a qualidade da assistência ${ }^{(14)}$.

A avaliação do processo da assistência pré-natal é importante para a melhoria da qualidade dos serviços e indispensável para promover a redução dos índices de mortalidade materna e perinatal, principalmente no Brasil, onde são observadas 
desigualdades sociais, econômicas, regionais e de acesso aos serviços de saúde. Essa avaliação constitui importante ferramenta que pode auxiliar na elaboração de políticas públicas mais efetivas e alinhadas à realidade territorial. A identificação de desigualdades é um aspecto fundamental do monitoramento e avaliação das políticas de atenção à saúde das mulheres.

Dentro desse contexto, este estudo tem como objetivo analisar o acesso à assistência pré-natal no Brasil a partir dos dados da Pesquisa Nacional de Saúde de 2013.

\section{MÉTODOS}

Estudo transversal descritivo com dados provenientes da PNS 2013, inquérito realizado pelo Instituto Brasileiro de Geografia e Estatística (IBGE) em parceria com o Ministério da Saúde (MS) e a Fundação Instituto Oswaldo Cruz (Fiocruz). A população-alvo do inquérito foi constituída por adultos ( $\geq 18$ anos de idade) residentes em domicílios particulares no território nacional. Não foram incluídos os setores censitários especiais (quartéis, bases militares, alojamentos, acampamentos, embarcações, penitenciárias, colônias penais, presídios, cadeias, asilos, orfanatos, conventos e hospitais).

O tamanho da amostra foi definido considerando-se o nível de precisão desejado para as estimativas de indicadores de interesse, sendo de 1.800 domicílios por unidade federativa. A PNS sorteou um total de 81.187 domicílios, sendo selecionado um indivíduo por domicílio. Após o encerramento da coleta, foram realizadas entrevistas em 64.348 domicílios, o que resultou em uma taxa de não resposta de $8,1 \%$.

Empregou-se o plano amostral por conglomerados com estratificação das unidades primárias de amostragem em três estágios. Os setores censitários ou conjunto de setores formam as unidades primárias de amostragem (UPAs), os domicílios representam as unidades de segundo estágio, e os moradores adultos definem as unidades de terceiro estágio.

Definiram-se pesos amostrais para as UPAs, para os domicílios e todos os seus moradores, além do peso para o morador selecionado, o qual foi calculado considerando-se o peso do domicílio correspondente, a probabilidade de seleção do morador, ajustes de não resposta por sexo, e calibração pelos totais populacionais por sexo e classes de idade, estimados com o peso de todos os moradores. Dividiu-se o questionário da PNS em módulos que contemplam as características do domicílio, de todos os moradores e do morador adulto selecionado ${ }^{(18)}$.

Através do Módulo S, a PNS investigou o atendimento pré-natal entre as mulheres de 18 a 49 anos de idade que tiveram algum parto no período de 01/01/2012 a 27/07/2013, considerando apenas o último parto para responder as questões.

No presente estudo, a variável "cobertura do pré-natal", foi definida pela proporção de mulheres que responderam positivamente ao questionamento: "Na última vez que a senhora esteve grávida, a senhora fez pré-natal?". Para esse grupo de mulheres, analisaram-se as variáveis: "proporção de gestantes com cartão pré-natal", "proporção de gestantes que iniciaram o pré-natal com menos de 13 semanas" e a "proporção de gestantes com todos os aconselhamentos".

O acesso à assistência pré-natal foi avaliado de acordo com os seguintes aspectos: as macrorregiões (Norte, Nordeste, Sul, Sudeste e Centro-Oeste), unidades da federação (26 estados e o Distrito Federal), faixa etária, cor da pele, nível de instrução, proporção de gestantes com exames solicitados/realizados (sangue, HIV, Sífilis, urina, ultrassonografia), aferição de peso, aferição da pressão arterial, medição do fundo de útero, ausculta do coração da criança, atendimento médico no parto, proporção de parto hospitalar, proporção de parto vaginal e presença de acompanhante no parto.

O presente estudo avaliou os dados da PNS que estavam disponíveis no site do DATASUS (www.datasus.gov.br) e as análises ocorreram entre dezembro de 2016 e janeiro de 2017. Os dados foram apresentados a partir de valores de proporções, média e intervalo de confiança a 95\%, avaliando-se a análise da significância entre os dados a partir da intercessão dos intervalos de confiança. Como se utilizaram somente os dados disponibilizados pelo Datasus, os pesquisadores só tiveram acesso aos valores relativos a cada evento, não tendo acesso aos valores absolutos.

O projeto da Pesquisa Nacional de Saúde recebeu aprovação pela Comissão Nacional de Ética em Pesquisa (CONEP), sob o n 10853812.7.0000.0008. O presente estudo utiliza dados secundários da PNS disponíveis em sites oficiais do Ministério da Saúde do Brasil, sendo dispensado de apreciação em comitê de ética em pesquisa em conformidade com a Resolução 4662012 do Conselho Nacional de Saúde.

\section{RESULTADOS}

A proporção de mulheres que afirmaram haver realizado pré-natal no Brasil foi de $97,4 \%$ ( $\left.\mathrm{IC}_{95 \%} 96,5-98,4 \%\right)$, sem diferenças entre o percentual de cobertura entre a área urbana e a área rural, nem entre as faixas etárias, embora tenha sido observada maior cobertura com o aumento da idade: de 18 a 29 anos $\left(97,07 \% \mathrm{IC}_{95 \%} 95,80-98,35\right)$, de 30 a 39 anos $\left(97,87 \% \mathrm{IC}_{95 \%} 96,39-99,35\right) \mathrm{e}$ de 40 a 49 anos $\left(99,05 \% \mathrm{IC}_{95 \%} 97,18-100,92\right)$.

Destaca-se que, em relação à cor da pele, as mulheres de cor preta tiveram cobertura menor $(93,78 \%$ IC95\% 88,94$98,62)$ do que em relação às pardas $(97,13 \%$ IC95\% 95,79-98,47) e brancas $(98,57 \%$ IC95\% 97,36-99,79). A cobertura do prénatal também variou de acordo com o grau de instrução, embora sem diferenças consideráveis: sem instrução e fundamental 
incompleto $(96,65 \%)$, fundamental completo e médio incompleto $(97,65 \%)$, médio completo e superior incompleto $(97,47 \%)$ e superior completo $(98,30 \%)$.

A região com menor cobertura de pré-natal foi a região Norte $\left(95,4 \% \mathrm{IC}_{95 \%} 95,4\right)$, seguida da região $\mathrm{Sul}_{(96,3} \mathrm{IC}_{95 \%} 92,7-$ $99,9)$, Centro-Oeste $\left(97,3 \% \mathrm{IC}_{95 \%} 95,0-99,7\right)$ e Sudeste $\left(97,8 \% \mathrm{IC}_{95 \%} 96,8-99,7\right)$. A região Nordeste foi a única com cobertura acima da média nacional $\left(98,2 \% \mathrm{IC}_{95 \%} 96,8-99,7\right)$. A região Norte e a Nordeste apresentaram uma média de 7 consultas/por gestante; a região Sudeste foi de 9 consultas/por gestante; e as regiões Centro-Oeste e Sul, de 10 ou mais consultas/por gestante.

Entre as gestantes que realizaram o pré-natal, $69,33 \%$ indicou que todas as consultas foram feitas através do SUS e para $3,84 \%$ indicou que algumas consultas foram feitas através do SUS. Das mulheres que realizaram o pré-natal pelo SUS, $60,52 \%$ realizou na unidade básica de saúde (posto/centro saúde/USF), 2,44\% em centro especialidades ou policlínica pública, e 8,93\% em hospital público/ambulatório. Para as demais mulheres (27,31\%), o pré-natal foi realizado em consultório particular ou clínica privada. Em 71,19\% dos casos, a consulta foi realizada pelo médico e, em $27,63 \%$, pelo enfermeiro.

Houve elevado percentual de mulheres que realizou pré-natal e recebeu o cartão pré-natal $\left(95,3 \% \mathrm{IC}_{95 \%} 93,6-97,0\right)$, sendo esse indicador considerado elevado para todas as regiões do país e não havendo diferença entre elas. Registrou-se, porém, baixa proporção de mulheres que receberam todos os aconselhamentos no período $\left(69,2 \% \mathrm{IC}_{95 \%} 65,5-72,9\right)$ (Figura 1). A proporção de "todos os aconselhamentos" é referente aos aconselhamentos para não faltar às consultas, ter uma alimentação saudável, não beber, não fumar e não usar tintura de cabelo.

Quanto à cobertura do pré-natal, as unidades da federação que registraram percentuais abaixo do valor nacional (97,40\% IC95\% 96,5-98,4\%) foram: Rondônia (88,2\%), Distrito Federal (91,3\%), Paraná (93,8\%), Amapá (93,3\%), Pará (94\%), Maranhão (94,9\%), Rio de Janeiro (94,9\%), Roraima (96,3\%), Minas Gerais (96,7\%), Paraíba (97\%), Mato Grosso do Sul $(97,1 \%)$ e Piauí $(97,1 \%)$.

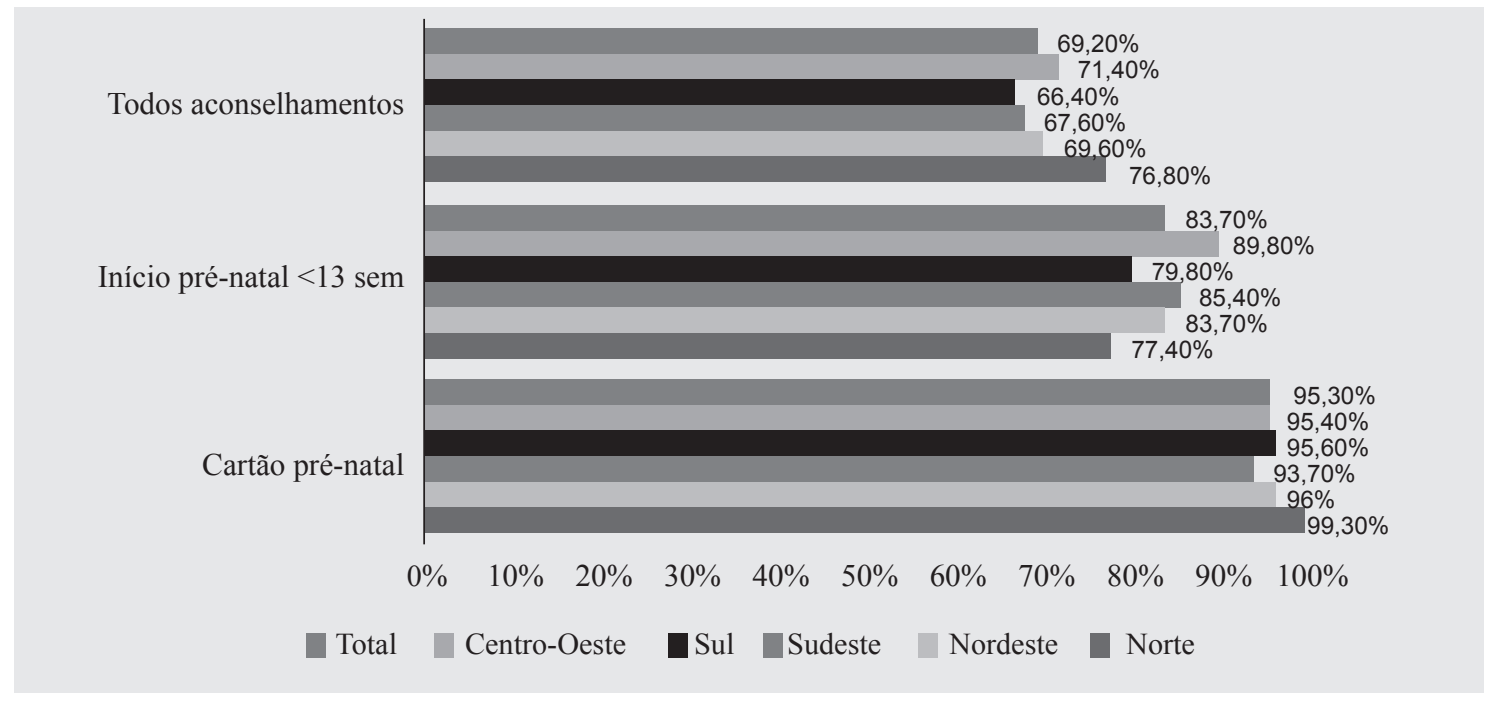

Figura 1 - Proporção de mulheres que realizaram o pré-natal com cartão pré-natal, iniciado com menos de 13 semanas e que receberam todos os aconselhamentos, por macrorregiões, Brasil, 2013.

Em relação à proporção de gestantes com cartão pré-natal, estão abaixo da média nacional (95,3\% IC95\% 93,6-97,0\%) os estados de Mato Grosso (83,6\%), Sergipe (84,2\%), Rio de Janeiro (90,1\%), Minas Gerais (93\%), Paraná (93,2\%), Bahia (94,1\%), Espírito Santo (94,2\%), Paraíba (94,5\%), São Paulo (94,9\%) e Alagoas (95\%). Para a proporção de mulheres com todos os aconselhamentos, as unidades federativas com valores inferiores à proporção nacional foram: Bahia (55,6\%), Minas Gerais (57\%), Maranhão (58,4\%), Rio de Janeiro (59,1\%), Goiás (63,3\%) e Acre $(68,1 \%)$ (Tabela I). 
Tabela I - Proporção de mulheres que realizaram o pré-natal e receberam todos os aconselhamentos e o cartão pré-natal por unidades da federação, Brasil, 2013.

\begin{tabular}{|c|c|c|c|}
\hline Unidade da federação & $\begin{array}{c}\text { Pré-natal } \\
\% \text { (IC 95\%) }\end{array}$ & $\begin{array}{l}\text { Aconselhamentos } \\
\% \text { (IC } 95 \% \text { ) }\end{array}$ & $\begin{array}{c}\text { Cartão pré-natal } \\
\% \text { (IC 95\%) }\end{array}$ \\
\hline \multirow{2}{*}{ Acre } & $100 \%$ & $68,1 \%$ & $97,8 \%$ \\
\hline & $(100-100 \%)$ & $(54,7-81,5 \%)$ & $(94,4-100,0 \%)$ \\
\hline \multirow{2}{*}{ Alagoas } & $97,80 \%$ & $69,7 \%$ & $95 \%$ \\
\hline & $(93,6-100 \%)$ & $(54,2-85,1 \%)$ & $(89,1-100,0 \%)$ \\
\hline Amapá & $93,30 \%$ & $87,6 \%$ & $100 \%$ \\
\hline \multirow{2}{*}{ Amazonas } & $\begin{array}{c}(87,2-99,4 \%) \\
98,60 \%\end{array}$ & $\begin{array}{c}(78,4-96,8 \%) \\
91,3 \%\end{array}$ & $\begin{array}{c}(100,0-100,0 \%) \\
99,8 \%\end{array}$ \\
\hline & $(96,8-100 \%)$ & $(84,6-97,9 \%)$ & $(99,3-100,0 \%)$ \\
\hline Bahia & $\begin{array}{c}99,20 \% \\
(97,8-100 \%)\end{array}$ & $\begin{array}{c}55,6 \% \\
(39,4-71,8 \%)\end{array}$ & $\begin{array}{c}94,1 \% \\
(87,4-100,0 \%)\end{array}$ \\
\hline Ceará & $\begin{array}{c}100 \% \\
(100-100 \%)\end{array}$ & $\begin{array}{c}75,1 \% \\
(63,2-87,1 \%)\end{array}$ & $\begin{array}{c}97,3 \% \\
(94,4-100,0 \%)\end{array}$ \\
\hline Distrito Federal & $\begin{array}{c}91,30 \% \\
(80,1-100 \%)\end{array}$ & $\begin{array}{c}81,4 \% \\
(69,3-93,5 \%)\end{array}$ & $\begin{array}{c}100 \% \\
(100,0-100,0 \%)\end{array}$ \\
\hline Espírito Santo & $\begin{array}{c}100 \% \\
(100-100 \%)\end{array}$ & $\begin{array}{c}76 \% \\
(61,1-90,8 \%)\end{array}$ & $\begin{array}{c}94,2 \% \\
(84,5-100,0 \%)\end{array}$ \\
\hline Goiás & $\begin{array}{c}98,70 \% \\
(96,8-100 \%)\end{array}$ & $\begin{array}{c}63,3 \% \\
(49,2-77,5 \%)\end{array}$ & $\begin{array}{c}96,9 \% \\
(93,1-100,0 \%)\end{array}$ \\
\hline Maranhão & $\begin{array}{c}94,90 \% \\
(86,9-100 \%)\end{array}$ & $\begin{array}{c}58,4 \% \\
(39,0-77,7 \%)\end{array}$ & $\begin{array}{c}98,1 \% \\
(95,5-100,0 \%)\end{array}$ \\
\hline Mato Grosso & $\begin{array}{c}100 \% \\
(100-100 \%)\end{array}$ & $74,1 \%(57,7-90,5 \%)$ & $\begin{array}{c}83,6 \% \\
(68,6-98,5 \%)\end{array}$ \\
\hline Mato Grosso do Sul & $\begin{array}{c}97,10 \% \\
(93,2-100 \%)\end{array}$ & $\begin{array}{c}81 \% \\
(68,3-93,7 \%)\end{array}$ & $\begin{array}{c}100 \% \\
(100,0-100,0 \%)\end{array}$ \\
\hline Minas Gerais & $\begin{array}{c}96,70 \% \\
(92,2-100 \%)\end{array}$ & $\begin{array}{c}57 \% \\
(38,2-75,8 \%)\end{array}$ & $\begin{array}{c}93 \% \\
(84,5-100,0 \%)\end{array}$ \\
\hline Pará & $\begin{array}{c}94 \% \\
(87,5-100 \%)\end{array}$ & $\begin{array}{c}69,6 \% \\
(56,4-82,7 \%)\end{array}$ & $\begin{array}{c}99 \% \\
(97,6-100,0 \%)\end{array}$ \\
\hline Paraíba & $\begin{array}{c}97 \% \\
(93,4-100 \%)\end{array}$ & $\begin{array}{c}88,9 \% \\
(79,3-98,5 \%)\end{array}$ & $\begin{array}{c}94,5 \% \\
(89,0-100,0 \%)\end{array}$ \\
\hline Paraná & $\begin{array}{c}93,80 \% \\
(86,6-100 \%)\end{array}$ & $\begin{array}{c}66,9 \% \\
(53,1-80,7 \%)\end{array}$ & $\begin{array}{c}93,2 \% \\
(85,1-100,0 \%)\end{array}$ \\
\hline Pernambuco & $\begin{array}{c}98,10 \% \\
(95,2-100 \%)\end{array}$ & $\begin{array}{c}81,4 \% \\
(71,5-91,2 \%)\end{array}$ & $\begin{array}{c}98 \% \\
(95,1-100,0 \%)\end{array}$ \\
\hline Piauí & $\begin{array}{c}9 /, 10 \% \\
(92,6-100 \%)\end{array}$ & $\begin{array}{c}80,3 \% \\
(66,0-94,6 \%)\end{array}$ & $\begin{array}{c}98,7 \% \\
(96,3-100,0 \%)\end{array}$ \\
\hline Rio de Janeiro & $\begin{array}{c}94,90 \% \\
(89,9-99,8 \%)\end{array}$ & $\begin{array}{c}59,1 \% \\
(46,1-72,1 \%)\end{array}$ & $\begin{array}{c}90,1 \% \\
(81,4-98,8 \%)\end{array}$ \\
\hline Rio Grande do Norte & $\begin{array}{c}100 \% \\
(100-100 \%)\end{array}$ & $\begin{array}{c}81 \% \\
(64,7-97,4 \%)\end{array}$ & $\begin{array}{c}100 \% \\
(100,0-100,0 \%)\end{array}$ \\
\hline Rio Grande do Sul & $\begin{array}{c}99,30 \% \\
(97,9-100 \%)\end{array}$ & $\begin{array}{c}77,5 \% \\
(65,6-89,4 \%)\end{array}$ & $\begin{array}{c}96,1 \% \\
(91,2-100,0 \%)\end{array}$ \\
\hline Rondônia & $\begin{array}{c}88,20 \% \\
(80-96,5 \%)\end{array}$ & $\begin{array}{c}74,5 \% \\
(61,3-87,6 \%)\end{array}$ & $\begin{array}{c}99,4 \% \\
(98,2-100,0 \%)\end{array}$ \\
\hline Roraima & $\begin{array}{c}96,30 \% \\
(92,2-100 \%)\end{array}$ & $\begin{array}{c}70,2 \% \\
(56,5-83,9 \%)\end{array}$ & $\begin{array}{c}100 \% \\
(100,0-100,0 \%)\end{array}$ \\
\hline Santa Catarina & $\begin{array}{c}98 \% \\
(94,9-100 \%)\end{array}$ & $\begin{array}{c}100 \% \\
(100,0-100,0 \%)\end{array}$ & $\begin{array}{c}100 \% \\
(100,0-100,0 \%)\end{array}$ \\
\hline São Paulo & $\begin{array}{c}98,80 \% \\
(97,5-100 \%)\end{array}$ & $\begin{array}{c}72,9 \% \\
(63,1-82,7 \%)\end{array}$ & $\begin{array}{c}94,9 \% \\
(89,8-99,9 \%)\end{array}$ \\
\hline Sergipe & $\begin{array}{c}100 \% \\
(100-100 \%)\end{array}$ & $\begin{array}{c}78,6 \% \\
(64,9-92,4 \%)\end{array}$ & $\begin{array}{c}84,2 \% \\
(70,4-98,1 \%)\end{array}$ \\
\hline Tocantins & $\begin{array}{c}100 \% \\
(100-100 \%)\end{array}$ & $\begin{array}{c}81 \% \\
(68,8-93,2 \%)\end{array}$ & $\begin{array}{c}100 \% \\
(100,0-100,0 \%)\end{array}$ \\
\hline Brasil & $\begin{array}{c}97,40 \% \\
(96,5-98,4 \%)\end{array}$ & $\begin{array}{c}69,2 \% \\
(65,5-72,9 \%)\end{array}$ & $\begin{array}{c}95,3 \% \\
(93,6-97,0 \%)\end{array}$ \\
\hline
\end{tabular}


Em relação ao acesso aos serviços de saúde no pré-natal, destacam-se as menores proporções de realização de teste sorológico para Sífilis $\left(64,8 \% \mathrm{IC}_{95 \%} 61,0-68,7 \%\right)$ e para o Vírus da Imunodeficiência Humana (HIV) $(88,8 \%$ IC $95 \%$ 86,3-91,3\%). (Tabela II).

Tabela II - Análise das variáveis de acesso a serviços de saúde no pré-natal de acordo com proporção de mulheres que realizaram pré-natal por região, Brasil, 2013.

\begin{tabular}{|c|c|c|c|c|c|c|}
\hline VARIÁVEIS & $\begin{array}{c}\text { Norte } \\
\%(\text { IC 95\%) }\end{array}$ & $\begin{array}{c}\text { Nordeste } \\
\% \text { (IC 95\%) }\end{array}$ & $\begin{array}{c}\text { Sudeste } \\
\% \text { (IC 95\%) }\end{array}$ & $\begin{array}{c}\text { Sul } \\
\%(\text { IC } 95 \%) \\
\end{array}$ & $\begin{array}{l}\text { Centro-oeste } \\
\%(\text { IC } 95 \%) \\
\end{array}$ & $\begin{array}{c}\text { Total } \\
\% \text { (IC 95\%) }\end{array}$ \\
\hline Aferição de peso & $\begin{array}{c}86,60 \% \\
(81,6-91,6 \%)\end{array}$ & $\begin{array}{c}88,5 \% \\
(83,7-93,2 \%)\end{array}$ & $\begin{array}{c}95,5 \%(93,4- \\
97,6 \%)\end{array}$ & $\begin{array}{c}93,5 \%(88,7- \\
98,3 \%)\end{array}$ & $\begin{array}{c}91,3 \%(86,5- \\
96,1 \%)\end{array}$ & $\begin{array}{c}91,9 \%(90,1- \\
93,8 \%)\end{array}$ \\
\hline $\begin{array}{l}\text { Aferição da pressão } \\
\text { arterial }\end{array}$ & $\begin{array}{c}87,6 \%(83,0- \\
92,1 \%)\end{array}$ & $\begin{array}{c}88,5 \%(83,8- \\
93,3 \%)\end{array}$ & $\begin{array}{c}93,7 \%(91,0- \\
96,4 \%)\end{array}$ & $\begin{array}{c}96,8 \%(94,3- \\
99,3 \%)\end{array}$ & $\begin{array}{c}93,4 \%(89,9- \\
96,9 \%)\end{array}$ & $\begin{array}{c}92 \%(90,2- \\
93,9 \%)\end{array}$ \\
\hline $\begin{array}{l}\text { Medição do fundo do } \\
\text { útero }\end{array}$ & $\begin{array}{c}91,4 \%(84,8- \\
98,1 \%)\end{array}$ & $\begin{array}{c}92,6 \%(87,7- \\
97,5 \%)\end{array}$ & $\begin{array}{c}97,7 \%(95,6- \\
99,8 \%)\end{array}$ & $\begin{array}{c}95,7 \%(91,1- \\
100,0 \%)\end{array}$ & $\begin{array}{c}98 \%(96,0- \\
100,0 \%)\end{array}$ & $\begin{array}{c}95,3 \% \\
(93,4-97,3 \%)\end{array}$ \\
\hline Ausculta coração do bebê & $\begin{array}{c}96,8 \%(93,4- \\
100,0 \%)\end{array}$ & $\begin{array}{c}93,5 \% \\
(88,7-98,4 \%)\end{array}$ & $\begin{array}{c}99,4 \%(98,5- \\
100,0 \%)\end{array}$ & $\begin{array}{c}100 \%(100,0- \\
100,0 \%)\end{array}$ & $\begin{array}{c}97,7 \%(95,5- \\
100,0 \%)\end{array}$ & $\begin{array}{c}97,4 \%(95,8- \\
98,9 \%)\end{array}$ \\
\hline PA elevada & $\begin{array}{c}18,7 \%(13,8- \\
23,7 \%)\end{array}$ & $\begin{array}{c}17,8 \% \\
(13,6-22,1 \%)\end{array}$ & $\begin{array}{c}19,1 \%(12,8- \\
25,3 \%)\end{array}$ & $\begin{array}{c}18,9 \%(11,1- \\
26,7 \%)\end{array}$ & $\begin{array}{c}24,4 \%(16,4- \\
32,3 \%)\end{array}$ & $\begin{array}{c}19,1 \%(16,0- \\
22,1 \%)\end{array}$ \\
\hline Exame de sangue & $\begin{array}{c}95,6 \%(92,9- \\
98,2 \%)\end{array}$ & $\begin{array}{c}97 \%(94,8- \\
99,2 \%)\end{array}$ & $\begin{array}{c}97,9 \%(96,0- \\
99,7 \%)\end{array}$ & $\begin{array}{c}96,4 \%(92,9- \\
99,9 \%)\end{array}$ & $\begin{array}{c}98,8 \%(97,4- \\
100,0 \%)\end{array}$ & $\begin{array}{c}97,3 \%(96,2- \\
98,4 \%)\end{array}$ \\
\hline Exame de Sífilis & $\begin{array}{c}69,5 \%(62,0- \\
77,1 \%)\end{array}$ & $\begin{array}{c}56,9 \%(50,1- \\
63,7 \%)\end{array}$ & $\begin{array}{c}63,7 \%(56,1- \\
71,3 \%)\end{array}$ & $\begin{array}{c}74,1 \%(66,0- \\
82,2 \%)\end{array}$ & $\begin{array}{c}76,1 \%(68,9- \\
83,2 \%)\end{array}$ & $\begin{array}{c}64,8 \%(61,0- \\
68,7 \%)\end{array}$ \\
\hline Exame de HIV & $\begin{array}{c}90,6 \%(86,6- \\
94,6 \%)\end{array}$ & $\begin{array}{c}84,1 \%(78,9- \\
89,4 \%)\end{array}$ & $\begin{array}{c}89,5 \%(85,0- \\
94,1 \%)\end{array}$ & $\begin{array}{c}93,5 \%(89,5- \\
97,5 \%)\end{array}$ & $\begin{array}{c}91,2 \%(86,6- \\
95,8 \%)\end{array}$ & $\begin{array}{c}88,8 \%(86,3- \\
91,3 \%)\end{array}$ \\
\hline Exame de urina & $\begin{array}{c}96,2 \%(93,3- \\
99,2 \%)\end{array}$ & $\begin{array}{c}97,1 \%(95,0- \\
99,2 \%)\end{array}$ & $\begin{array}{c}98,9 \%(97,8- \\
100,0 \%)\end{array}$ & $\begin{array}{c}99,2 \%(98,1- \\
100,0 \%)\end{array}$ & $\begin{array}{c}98,1 \%(96,3- \\
99,9 \%)\end{array}$ & $\begin{array}{c}98,1 \%(97,3- \\
98,9 \%)\end{array}$ \\
\hline Ultrassonografia & $\begin{array}{c}94,6 \%(91,8- \\
97,4 \%)\end{array}$ & $\begin{array}{c}96,4 \%(92,2- \\
100,0 \%) \\
\end{array}$ & $\begin{array}{c}98,8 \%(97,8- \\
99,8 \%)\end{array}$ & $\begin{array}{c}99 \%(97,6- \\
100,0 \%) \\
\end{array}$ & $\begin{array}{c}99 \%(98,0- \\
100,0 \%) \\
\end{array}$ & $\begin{array}{c}97,7 \%(96,4- \\
99,1 \%)\end{array}$ \\
\hline
\end{tabular}

PA: Pressão arterial

$\mathrm{Na}$ assistência ao parto, $10,42 \%$ das gestantes $\left(\mathrm{IC}_{95 \%} 8,2-12,5 \%\right)$ procuraram mais de 01 estabelecimento de saúde, tendo ocorrido com 9,4\% ( $\left.\mathrm{IC}_{95 \%} 5,8-13,0 \%\right)$ das gestantes na região Norte e 16,4\% $\left(\mathrm{IC}_{95 \%} 11,8-21,0\right)$ na região Nordeste. Destaca-se a proporção de $45,3 \%\left(\mathrm{IC}_{95 \%} 1,4-49,3 \%\right)$ de partos vaginais, com maior proporção nas regiões Norte $\left(59,8 \% \mathrm{IC}_{95 \%} 52,8-66,8\right)$ Nordeste $\left(55 \% \mathrm{IC}_{95 \%} 48,9-61,2 \%\right)$ (Tabela III).

Tabela III - Análise as variáveis de acesso a serviços de saúde na assistência ao parto de acordo com proporção de mulheres que realizaram pré-natal por região, Brasil, 2013.

\begin{tabular}{lcccccc}
\hline VARIÁVEIS & $\begin{array}{c}\text { Norte } \\
\text { \% (IC95\%) }\end{array}$ & $\begin{array}{c}\text { Nordeste } \\
\text { \% (IC95\%) }\end{array}$ & $\begin{array}{c}\text { Sudeste } \\
\text { \% (IC95\%) }\end{array}$ & $\begin{array}{c}\text { Sul } \\
\text { \% (IC95\%) }\end{array}$ & $\begin{array}{c}\text { Centro-oeste } \\
\text { \% (IC95\%) }\end{array}$ & $\begin{array}{c}\text { Total } \\
\text { \% (IC95\%) }\end{array}$ \\
\hline Atendimento médico & $76,1 \%(68,5-$ & $78 \%$ & $93,8 \%(90,9-$ & $93,2 \%$ & $94,6 \%$ & $87,4 \%(84,9-$ \\
no parto & $83,7 \%)$ & $(71,6-84,5 \%)$ & $96,6 \%)$ & $(88,6-97,9 \%)$ & $(91,1-98,1 \%)$ & $89,9 \%)$ \\
Parto hospitalar & $95,9 \%(93,6-$ & $96,6 \%$ & $98,5 \%(97,3-$ & $99,9 \%(99,7-$ & $97,8 \%(95,4-$ & $97,9 \%(97,0-$ \\
& $98,2 \%)$ & $(94,3-99,0)$ & $99,7 \%)$ & $100,0 \%)$ & $100,0 \%)$ & $98,7 \%)$ \\
Parto vaginal & $59,8 \%(52,8-$ & $55 \%$ & $37,4 \%(30,0-$ & $38,3 \%$ & $42,4 \%$ & $45,3 \%(41,4-$ \\
\multirow{2}{*}{ Acompanhante } & $66,8 \%)$ & $(48,9-61,2 \%)$ & $44,8 \%)$ & $(27,7-48,9 \%)$ & $(34,4-50,4 \%)$ & $49,3 \%)$ \\
parto & $50 \%$ & $55,9 \%(46,1-$ & $59,4 \%$ & $77,2 \%(66,1-$ & $42,8 \%(31,0-$ & $58 \%(52,3-$ \\
Parto & $(38,3-$ & $65,8 \%)$ & $(47,5-$ & $88,2 \%)$ & $54,6 \%)$ & $63,7 \%)$ \\
marcado & $61,6 \%)$ & $71,2 \%)$ & $47,7 \%$ & $50,2 \%$ & $53,5 \%(48,4-$ \\
\hline
\end{tabular}

\section{DISCUSSÃO}

Os resultados do presente estudo demonstram que, apesar da elevada cobertura da assistência pré-natal no Brasil, ainda há inadequações no serviço, revelados pelos indicadores de proporção de aconselhamentos (orientações) e de acesso à realização de exames laboratoriais, além das disparidades da cobertura de alguns serviços entre as regiões brasileiras. 
Após a implantação do Programa de Humanização do Pré-Natal e Nascimento (PHPN), criado no ano de 2000 pelo Ministério da Saúde, a qualidade da assistência pré-natal tem sido objeto de estudo em várias regiões do país ${ }^{(18)}$. A elevação da cobertura da assistência pré-natal no Brasil vem sendo observada desde os anos 1990, e alcança atualmente valores superiores a $90 \%$ em todas as regiões do país, abrangendo mulheres com diferentes características demográficas, sociais e reprodutivas ${ }^{(12,}$ 14,19-21), em concordância com os dados da pesquisa.

Estudo nacional de base hospitalar com puérperas e seus recém-nascidos, a pesquisa Nascer no Brasil traz dado de cobertura de assistência pré-natal de $98,7 \%{ }^{(10)}$, semelhante aos $97,4 \%$ desse estudo, número que deixa evidente o avanço e manutenção da cobertura superior a $90 \%$ no país. Todavia, observa-se que não há um consenso para esses achados. A partir de uma revisão de estudos brasileiros realizados entre 2005 a 2015, observou-se que a maioria dos estudos encontrou baixa qualidade do prénatal, com níveis de cobertura variando em geral de 4,5 a $66,1 \%{ }^{(12)}$ e com baixo percentual de adequação de acordo com as recomendações do Programa de Humanização do Pré-natal e Nascimento, do Ministério da Saúde ${ }^{(14,19-22)}$.

No entanto, um menor acesso à assistência pré-natal ainda ocorre para mulheres indígenas e pretas e para aquelas com menor escolaridade, com maior número de gestações e para as residentes nas regiões Norte ${ }^{(12,17)}$ e Nordeste. Esses dados de acesso são concordantes com o presente estudo, o que deixa evidente a persistência de desigualdades sociais no acesso a esse serviço de saúde fundamental ${ }^{(17)}$. Um estudo transversal realizado com 795 puérperas atendidas em unidades básicas de saúde de Santa Maria, Rio Grande do Sul, Brasil ${ }^{(23)}$, mostrou que mulheres com menor renda familiar, menor escolaridade e não brancas geralmente ingressam tardiamente no pré-natal, e que a assistência a essas mulheres tem qualidade inferior a que é prestada para mulheres que não possuem essas características.

A assistência realizada no primeiro trimestre constitui um indicador da qualidade dos cuidados maternos ${ }^{(6)}$. Os dados da PNS revelam que a região Norte apresenta os menores valores do início do pré-natal com menos de 13 semanas de gestação, confirmando as profundas desigualdades no acesso aos serviços de saúde entre as regiões brasileiras. Estudo com dados de assistência pré-natal de São Luís, Maranhão, relatou que apenas 39,8\% da amostra estudada teve assistência adequada ${ }^{(15)}$. Por outro lado, uma coorte retrospectiva realizada em Londrina, Paraná revelou que 91,4\% das mulheres incluídas realizaram prénatal, e destas, $71,7 \%$ iniciaram o pré-natal no primeiro trimestre ${ }^{(16)}$. A partir da PNS, observou-se que $83,7 \%$ das gestantes iniciaram o pré-natal no primeiro trimestre. O início tardio do acompanhamento pré-natal evidencia a fragilidade na organização dos serviços de saúde e as lacunas no processo de trabalho de captação precoce de gestantes e de conscientização da população( ${ }^{(23)}$.

Quanto às desigualdades regionais relativas ao número de consultas de pré-natal, os achados do presente estudo estão em consonância com os relatórios do Ministério da Saúde ${ }^{(23)}$, que enfatizam que, nas regiões Sudeste e Sul, $83 \%$ das mulheres realizam seis ou mais consultas no pré-natal, enquanto nas regiões Norte e Nordeste esse número cai para $57 \%$ e $67 \%$, respectivamente, com maior proporção de mulheres que declararam ter realizado três consultas ou menos nessas regiões (Norte com $21 \%$ e Nordeste com 14,1\%). Em um inquérito transversal em áreas pobres das regiões Norte e Nordeste do Brasil, visando avaliar a assistência à gestação e ao parto entre mães de menores de cinco anos, os resultados apontaram que existe uma cobertura assistencial insuficiente devido às baixas condições sociais e dificuldades de acesso nessas regiões, tornando mais tardia a implementação de novos programas e tecnologias nesse tipo de assistência ${ }^{(21)}$.

Essa discrepância regional entre o número de consultas de pré-natal pode ser reflexo das desigualdades do acesso aos sistemas de saúde. Considerando a recomendação de mínimo de oito consultas pré-natais, o baixo número de consultas impacta o processo de prevenção e/ou deteç̧ão precoce de patologias, tanto maternas quanto fetais, podendo incidir em aumento dos números de casos de mortalidade materna ou perinatal. Além disso, o baixo número de consultas prejudica o processo de informação e orientações essenciais aos cuidados durante a gestação e o puerpério, criando um ciclo de desinformação que pode ser prejudicial ${ }^{(4)}$.

Durante a assistência pré-natal, é requisito obrigatório o cartão pré-natal para registro de todas as consultas, exames e procedimentos realizados ${ }^{(6)}$. Quanto à proporção de gestantes com cartão pré-natal, a região Nordeste apresenta os menores valores frente à média nacional, seguida pela região Sudeste, resultado que está de acordo com os achados do estudo realizado com os dados da Pesquisa Nascer no Brasil, realizado entre 2011 e 2012, que afirma que mais de $90 \%$ das mulheres receberam o cartão de pré-natal, todavia com menores proporções entre as regiões Norte e Centro-Oeste ${ }^{(12)}$. Entretanto a pesquisa que analisou a assistência pré-natal em municípios do Nordeste do Brasil( ${ }^{(2)}$ e verificou a distribuição do cartão pré-natal em 31 municípios do Nordeste, constatou que apenas $74,2 \%$ das gestantes haviam tido acesso a esse instrumento de acompanhamento, mostrando que ainda há resultados divergentes entre os estudos que avaliam a assistência pré-natal no Brasil.

Recomenda-se que a gestante acompanhe o processo de gestação, sendo o cartão pré-natal uma ferramenta essencial que contém as informações clínicas e principais orientações para a gestante. A ausência ou preenchimento inadequado desse instrumento pode prejudicar a correta condução do acompanhamento da gestante e dificultar a tomada de decisão por parte dos profissionais envolvidos, principalmente nos casos de gestação de risco ${ }^{(4)}$.

Outro resultado que se destaca é a baixa proporção de aconselhamentos. É possível que isso se deva ao fato de que as orientações durante o período gestacional, apesar de serem essenciais, nem sempre são exploradas pelos profissionais ${ }^{(23)}$. No Brasil, a insuficiência das informações na assistência pré-natal é comum e gera insatisfação e desqualificação do atendimento 
recebido ${ }^{(25)}$. A falta de orientação durante as consultas, a não realização dos exames laboratoriais de rotina, os procedimentos básicos recomendados e falta de prescrições são fatores que contribuem para a baixa qualidade da atenção pré-natal ${ }^{(13)}$.

A deficiência de informação prestada pode estar atrelada a má condução dos cuidados essenciais e, em casos mais extremos, influenciar no número de ocorrências de prematuridade e mortalidade materna. Assim, é imprescindível que os profissionais estejam sempre atentos e sensíveis aos questionamentos das gestantes, além de dispostos a disponibilizar informações relacionadas principalmente aos cuidados durante a gestação, a amamentação e período perinatal. Esses aconselhamentos podem ser realizados individualmente ou através de grupos de educação em saúde, que podem ser organizados nos próprios serviços por equipes multiprofissionais, e impactarão positivamente na qualidade da gestação das usuárias atendidas, elevando o padrão do serviço prestado.

Em relação à menor proporção de exames realizados nas regiões Norte e Nordeste evidenciados nesse estudo, esses resultados encontram-se de acordo com os achados do estudo sobre assistência pré-natal no Brasil a partir dos dados da Pesquisa Nascer no Brasil, que também confirmam que a menor proporção de registro de todos os exames foi encontrada nessas regiões ${ }^{(12)}$.

O manejo dos fatores de risco modificáveis que surgem durante a gestação é de grande importância para a redução da mortalidade materna. Mesmo com tendência de redução entre os anos de 1990 e 2010, a principal causa específica de morte materna no Brasil foi a hipertensão arterial na gestação ${ }^{(26)}$. Considerando que a realização dos exames pré-natais é indispensável para garantir a boa condução da gestação e a deteç̧ão precoce de doenças que podem ocorrer nesse período, faz-se imprescindível que sejam realizados corretamente, em tempo oportuno e com qualidade diagnóstica.

Sobre os profissionais atuantes na assistência pré-natal, essa prática ainda está fundamentada na consulta médica, sendo delegado ao profissional de enfermagem as atividades burocráticas relacionadas ao agendamento de consultas médicas, exames, orientações sobre as prescrições médicas e inserção de informação, cadastro e controle no Sistema de Informação em Saúde (SIS - Pré-Natal) ${ }^{(4,25)}$. Apesar da maior proporção de pré-natal realizado por médicos, contudo, foi encontrada na presente pesquisa que cerca de $30 \%$ das participantes realizaram consultas com enfermeiro.

Em concordância com os resultados do presente estudo também estão os resultados de um estudo transversal realizado no Rio Grande do Sul com o objetivo de avaliar a assistência pré-natal oferecida no âmbito público e privado ${ }^{(27)}$, em que verificaram que a principal porta de entrada para gestantes é a Estratégia Saúde da Família na Atenção Básica, seguidas da assistência em clínicas ou consultórios particulares, demonstrando a expansão dos serviços públicos que oferecem assistência pré-natal vinculados ao Sistema Único de Saúde, todavia esses achados também mostram, nos indicadores relativos ao trimestre de início do pré-natal, realização de seis ou mais consultas, execução de exames laboratoriais e clínicos, orientações diversas, realização das consultas com o mesmo profissional médico e adequação do pré-natal, que houve vantagens na assistência particular.

No Brasil, o acesso da população aos diferentes níveis assistenciais é um dos sentidos atribuídos à integralidade. Embora o acesso universal garantido constitucionalmente tenha derrubado barreiras formais, dificuldades no acesso e na continuidade da atenção permanecem, tanto na atenção básica quanto nos serviços especializados ${ }^{(28)}$.

Em relação à assistência ao parto, o relatório do Ministério da Saúde cita o crescente número de cesáreas em todas as regiões do país entre 2000 e $2012^{(26)}$. O estudo cita também a menor proporção de parto cesáreo nas regiões Norte e Nordeste, que corrobora com os dados encontrados neste estudo. Essas regiões, porém, apresentaram aumentos na execução desse tipo de parto entre 2000 e 2012 ( $45 \%$ e 48\%, respectivamente), aproximando-se dos valores encontrados entre outras regiões (cerca de $60 \%$ ).

As regiões Norte e Nordeste ainda se mantêm com a maior taxa proporcional de partos normais, uma vez que, considerando as questões regionais relacionadas às dificuldades de acesso aos serviços de saúde e fatores culturais, ainda contemplam a presença de parteiras e do parto domiciliar, bem como a escolha individual na opção pelo parto normal, mantém esses números elevados. Podem ainda estar relacionados a esse achado o número de multíparas nessas regiões, o que facilita o processo de escolha pelo parto normal, associado à dificuldade de acesso aos profissionais e à estrutura física necessárias para o parto cesáreo $^{(21,24,26)}$.

No presente estudo, o delineamento transversal não permitiu a utilização da temporalidade como critério de causalidade, uma vez que fatores de risco e desfecho foram aferidos ao mesmo tempo e o viés da causalidade reversa não pode ser eliminado, e isso constitui uma das limitações desse estudo. O tema abordado é de natureza pessoal, relacionada à saúde reprodutiva da mulher, além da ocorrência do viés de memória, que podem ter influenciado os resultados. Entretanto, o tamanho amostral alcançado e os corretos procedimentos de pesquisa adotados fortalecem a confiabilidade dos dados. Por se tratar de um inquérito populacional, este estudo permitiu a identificação da cobertura real do pré-natal no Brasil.

\section{CONCLUSÃO}

A assistência pré-natal no Brasil alcançou cobertura praticamente universal, mas persistem desigualdades no acesso ao cuidado adequado, que teria potencial de reverter os indicadores de morte materna, neonatal e perinatal ainda observados no país. Estratégias voltadas para as populações socialmente menos favorecidas são essenciais para facilitar o ingresso precoce no pré-natal e o contato com os serviços de saúde, garantindo a realização de cuidados efetivos em saúde. 
A ampliação do acesso à atenção primária para o início precoce da assistência pré-natal e a melhor utilização dos contatos com os serviços de saúde para a realização de cuidados efetivos em saúde, tanto em atendimentos individuais quanto coletivos, revelam-se prioritários na reversão desse quadro.

\section{CONFLITO DE INTERESSES}

Os autores declaram não haver conflito de interesse, incluindo interesses financeiros específicos, de relacionamentos e afiliações relevantes ao tema, ou sobre materiais discutidos no manuscrito.

\section{REFERÊNCIAS}

1. Souza JP. Mortalidade materna no Brasil: a necessidade de fortalecer os sistemas de saúde. Rev Bras Ginecol Obstet. 2011;33(10):273-9.

2. Ferraz L, Bordignon M. Mortalidade materna no Brasil: uma realidade que precisa melhorar. Rev Baiana Saúde Pública. 2013;36(2):527-38.

3. World Health Organization. Trends in maternal mortality: 1990 to 2013. Estimates by WHO, UNICEF, UNFPA, The World Bank and the United Nations Population Division: Geneva: WHO; 2014.

4. World Health Organization. WHO recommendations on antenatal care for a positive pregnancy experience. Geneva: WHO; 2016.

5. World Health Organization. The Millennium Development Goals Report 2008 [acesso em 2017 Jan 7]. Disponível em: http://www.un.org/millenniumgoals

6. Ministério da Saúde (BR), Secretaria de Atenção à Saúde, Departamento de atenção básica. Atenção ao pré-natal de baixo risco. Brasília: Ministério da Saúde; 2013.

7. Morse ML, Fonseca SC, Barbosa MD, Calil MB, Eyer FPC. Mortalidade materna no Brasil: o que mostra a produção científica nos últimos 30 anos?. Cad Saúde Pública. 2011;27(4):623-38.

8. Brasil. Portaria $\mathrm{N}^{\circ}$ 1.459, de 24 de junho de 2011. Institui no âmbito do Sistema Único de Saúde - SUS - a Rede Cegonha [acesso em 2017 Jan 7]. Disponível em: http://bvsms.saude.gov.br/bvs/saudelegis/gm/2011/prt1459_24_06_2011.html

9. Brasil. Portaria $\mathrm{n}^{\circ} 569$, de $1^{\circ}$ de Junho de 2000. Institui o Programa de Humanização no Pré-natal e Nascimento no âmbito do Sistema Único de saúde [acesso em 2017 Jan 7]. Disponível em: http://bvsms.saude.gov.br/bvs/saudelegis/gm/2000/ prt0569_01_06_2000_rep.html

10. Serruya SJ, Cecatti JG, do Lago TDG. O Programa de Humanização no Pré-natal e Nascimento do Ministério da Saúde no Brasil: resultados iniciais. Cad Saúde Pública. 2004;20(5):1281-9.

11. Polgliane RBS, Leal MC, Amorim MH, Zandonade E, Santos ET Neto. Adequação do processo de assistência pré-natal segundo critérios do Programa de Humanização do Pré-natal e Nascimento e da Organização Mundial de Saúde. Ciênc Saúde Coletiva. 2014;19(7):1999-2010.

12. Viellas EF, Domingues RMSM, Dias MAB, Gama SGN, Theme MM Filha, da Costa JV, et al. Assistência pré-natal no Brasil. Cad Saúde Pública. 2014;30(Sup 10):S85-S100.

13. Nunes JT, Rejane K, Gomes O, Thaís M, Rodrigues P, Denis M, et al. Quality of prenatal care in Brazil: review of published papers from 2005 to 2015. Cad Saúde Coletiva. 2016; 24(123):252-61.

14. Domingues RMSM, Hartz ZMA, Dias MAB, Leal MC. Avaliação da adequação da assistência pré-natal na rede SUS do Município do Rio de Janeiro, Brasil. Cad Saúde Pública. 2012;28(3):425-37.

15. Goudard MJF, Simões VMF, Batista RFL, Queiroz RCDS, Alves MTSSD, Coimbra LC, et al. Inadequacy of the content of prenatal care and associated factors in a cohort in the northeast of Brazil. Ciênc Saúde Coletiva. 2016;21(4):1227-38.

16. Ferrari RAP, Bertolozzi MR, Dalmas JC, Girotto E. Associação entre assistência pré-natal e mortes neonatais, 2000-2009, Londrina-PR. Rev Bras Enferm. 2014;67(3):354-9.

17. Domingues RMSM, Viellas EF, Dias MAB, Torres JA, Theme-Filha MM, Gama SGN, et al. Adequação da assistência prénatal segundo as características maternas no Brasil. Rev Panam Salud Publica. 2015;37(3):140-7

18. Damacena GN, Szwarcwald CL, Malta DC, Souza PRB Júnior, Vieira MLFP, Pereira CA, et al . O processo de desenvolvimento da Pesquisa Nacional de Saúde no Brasil, 2013. Epidemiol Serv Saúde. 2015;24(2):197-206. 
19. Andreucci CB, Cecatti JG. Desempenho de indicadores de processo do Programa de Humanização do Pré-natal e Nascimento no Brasil: uma revisão sistemática. Cad Saúde Pública. 2011;27(6):1053-64.

20. Coutinho T, Monteiro MFG, Sayd JD, Teixeira MTB, Coutinho CM, Coutinho LM. Monitoring the prenatal care process among users of the Unified Health Care System in a city of the Brazilian Southeast. Rev Bras Ginecol Obstet. 2010;32(11):563-9.

21. Chrestani MAD, Santos IS, Cesar JA, Winckler LS, Gonçalves TS, Neumann NA. Assistência à gestação e ao parto: resultados de dois estudos transversais em áreas pobres das regiões Norte e Nordeste do Brasil. Cad Saúde Pública. 2008;24(7):1609-18.

22. Nascimento LFC. Perfil de gestantes atendidas nos períodos pré-natal e perinatal: estudo comparativo entre os serviços público e privado em Guaratinguetá, São Paulo. Rev Bras Saúde Matern Infant. 2003;3(2):187-94.

23. Anversa ETR, Bastos GAN, Nunes LN, Pizzol TSD. Qualidade do processo da assistência pré-natal: unidades básicas de saúde e unidades de Estratégia Saúde da Família em município no Sul do Brasil. Cad Saúde Pública. 2012;28(4):789-800.

24. Rocha AF. Mortalidade neonatal: assistência pré-natal em municípios do Nordeste do Brasil [mestrado]. Rio de Janeiro: Escola Nacional de Saúde Pública Sergio Arouca; 2013.

25. Lamy GO, Moreno BS. Assistência pré-natal e preparo para o parto. Omnia Saúde. 2013;10(2):19-35.

26. Ministério da Saúde (BR), Secretaria de Vigilância em Saúde. Mortalidade materna no Brasil. Brasília: Ministério da Saúde; 2012. (Boletim 1/2012).

27. Cesar JA, Sutil AT, Santos GB, Cunha CF, Mendoza-Sassi RA. Assistência pré-natal nos serviços públicos e privados de saúde: estudo transversal de base populacional em Rio Grande, Rio Grande do Sul, Brasil. Cad Saúde Pública. 2012;28(11):2106-14.

28. Brito-Silva K, Bezerra AFB, Chaves LDP, Tanaka OY. Integralidade no cuidado ao câncer do colo do útero: avaliação do acesso. Rev Saúde Pública. 2014;48(2):240-8.

\section{Endereço para correspondência:}

Aryelly Dayane da Silva Nunes

Universidade Federal do Rio Grande do Norte - UFRN

Programa de Pós-Graduação em Saúde Coletiva

Av. Sen. Salgado Filho, 1787

Bairro: Lagoa Nova

CEP 59056-000 - Natal - RN - Brasil

E-mail: aryellydayane@gmail.com 